\title{
Estudio PETRO: evaluación de tromboprofilaxis y riesgo embólico en pacientes hospitalizados en una institución de tercer nivel en Bogotá, Colombia
}

\author{
PETRO study: assessment of thromboprophylaxis \\ and embolic risk in hospitalized patients at a \\ third-level institution in Bogota, Colombia
}

\author{
Oscar Alberto Sáenz Morales, MD. (1); Erika Vergara Vela, MD. (2); Francisco González \\ Acosta, MD., MSc. ${ }^{(3)}$; PAOLA BonILlA GonZÁlez, MD. ${ }^{(4)}$; JoRge ANDRÉS RuSSI LOZANO, MD. ${ }^{(5)}$; \\ AngÉlica Rojas DuRÁn, MD. ${ }^{(6)}$; Martín Zapata Laguado, MD. ${ }^{(7)}$; CAMILO Uhía GonzÁlez, MD. ${ }^{(8)}$
}

\section{Resumen}

Teniendo en cuenta que la tromboembolia venosa es la mayor causa de muerte evitable en el paciente hospitalizado, se hizo un estudio de tromboprofilaxis, tipo descriptivo retrospectivo, de serie de casos, en pacientes médicos y quirúrgicos hospitalizados en el servicio de urgencias. Se tuvieron en cuenta las indicaciones dadas por las principales escalas de riesgo embólico. Se utilizó base de datos en Excel y programa SPSS versión 23. En la mayoría de casos se utilizó tromboprofilaxis farmacológica con enoxaparina; se encontró profilaxis para riesgo bajo en $69 \%$, en medio $81 \%$ y en alto $92 \%$. La tasa de eventos adversos fue baja. Esta terapia presentó bajo costo, seguridad y eficacia. Se debe continuar mejorando la tromboprofilaxis implementando estrategias como alarmas, adherencias a guías, protocolos y educación continua.

Palabras clave: tromboprofilaxis, tromboembolia venosa, escalas de riesgo, enoxaparina, eficacia.

\begin{abstract}
Aware of the fact that venous thromboembolism is the leading cause of death in hospitalized patients, we carried out a study of thromboprophylaxis. The study was of the retrospective type, a case series of medical and surgical patients hospitalized in the emergency ward. We took into account the indications given by the main scales of embolic risk. We used an Excel database and the SPSS 23 software. In most cases, thromboprophylaxis with enoxaparin was used; we found prophylaxis for low risk in $69 \%$, for medium risk in $81 \%$, and for high risk in $92 \%$. The rate of adverse effects was low. This therapy proved to be cost-efficient, safe, and effective. It is necessary to further improve thromboprophylaxis by implementing strategies such as alarms, adherence to guidelines, protocols, and continued education.
\end{abstract}

Keywords: thromboprophylaxis, venous thromboembolism, risk scales, enoxaparin, efficacy.

\footnotetext{
${ }^{(1)}$ Médico Internista, Neumólogo, Grupo Investigación Urgencias Hospital Santa Clara (HSC), Coordinador Servicio de Urgencias HSC. Bogotá, Colombia.

${ }^{(2)}$ Médica Internista, Epidemióloga, Hospital Santa Clara (HSC). Docente Universidad El Bosque. Grupo Investigación Urgencias Hospital Santa Clara (HSC). Bogotá, Colombia.

${ }^{(3)}$ Médico Farmacólogo, Bioeticista, Hospital Santa Clara. Docente titular Universidad El Bosque. Grupo Investigación Urgencias Hospital Santa Clara (HSC). Bogotá, Colombia.

(4) Médica Internista, Hospital Santa Clara. Docente Universidad El Bosque, Grupo Investigación Urgencias Hospital Santa Clara (HSC). Bogotá, Colombia.

${ }^{(5)}$ Médico Internista, Epidemiólogo, Hospital Santa Clara. Docente Universidad El Bosque. Grupo Investigación Urgencias Hospital Santa Clara (HSC). Bogotá, Colombia.

${ }^{(6)}$ Médica Internista, Hospital Santa Clara. Docente Universidad El Bosque. Grupo Investigación Urgencias Hospital Santa Clara (HSC). Bogotá, Colombia.

${ }^{(7)}$ Residente segundo año Medicina Interna, Universidad el Bosque, Hospital Santa Clara. Bogotá, Colombia.

${ }^{\left({ }^{8}\right)}$ Residente tercer año Medicina Interna, Universidad El Bosque, Hospital Santa Clara. Bogotá, Colombia.

Correspondencia: Oscar Alberto Sáenz Morales, correo electrónico: oasm15@ yahoo.com

Recibido: 10/12/16. Aceptado: 10/01/17.
} 


\section{Introducción}

En el año 1856, Virchow hizo las primeras aproximaciones sobre la génesis de la enfermedad tromboembólica, creando los términos de trombosis y embolia. A la vez planteó y justificó que la presencia de un coágulo en las arterias o venas pulmonares no se origina en sí en este sistema vascular, sino que en cambio tiene un origen más distal probablemente en el sistema vascular periférico $(1,2)$.

Las nuevas masas de coágulo se depositan en la sangre y se van añadiendo capa tras capa; este crecimiento va formando una especie de cilindro inestable que se vuelve la fuente real de peligro que al migrar lleva a oclusión de los vasos remotos. Así se define como una regla que los trombos desde la periferia del cuerpo producen obstrucciones secundarias y depósitos metastásicos a los pulmones (2).

A este fenómeno se le conoce en la actualidad, como tríada de Virchow, que contiene la estasis venosa, el daño endotelial y la hipercoagulabilidad; este término es usado para describir la etiología y analizar el riesgo de trombosis especialmente en trombosis venosa profunda $(1,3)$.

Acudiendo a estos mecanismos fisiopatológicos, en múltiples escenarios de pacientes hospitalizados, se ha encontrado un aumento de la incidencia de complicaciones tromboembólicas, con tasas de mortalidad que oscilan entre 5 a $10 \%$ secundarias a embolia pulmonar (4); 18,3\% de incidencia de tromboembolia venosa (5).

La tromboembolia abarca dos condiciones relacionadas entre sí que forman parte del mismo espectro: la trombosis venosa profunda y la embolia pulmonar; esta última es la obstrucción del flujo sanguíneo a una o más arterias del pulmón por un trombo alojado en un vaso pulmonar.

Con el fin de ir a la vanguardia de los nuevos acontecimientos que amenazan la salud del paciente en la estancia hospitalaria se buscan varias medidas para prevenir complicaciones tales como trombosis venosa profunda en los miembros inferiores, tromboembolia pulmonar y ataque cerebro vascular (6), clasificadas como potencialmente letales y prevenibles durante la estancia hospitalaria, perioperatoria y postoperatoria, en paciente médico y quirúrgico.

Estas complicaciones se convierten en una de las principales causas de discapacidad a largo plazo o de muerte para los pacientes hospitalizados, y generan mayores costos para el sistema de salud con relación al uso oportuno y adecuado de la tromboprofilaxis. Por ello, la Organización Mundial de la Salud, por medio de la Alianza Mundial para la seguridad del paciente y su consenso de expertos internacionales, ha incluido la enfermedad tromboembólica dentro de las "lesiones debidas a errores quirúrgicos, médicos y anestésicos", como uno de los 23 tópicos con substancial impacto sobre la seguridad del paciente y que requiere atención urgente, además de ser considerada como una resultante de cuidado médico inseguro (7). Se considera, por ende, que con una tromboprofilaxis adecuada se previene de forma más eficiente este desenlace fatal para el paciente.

\section{Fisiopatología de la trombosis}

El trombo es un fenómeno patológico que se aloja en venas, arterias y cavidades cardiacas, generando trombosis, proceso que causa la obstrucción del flujo sanguíneo evidenciado en los tejidos y células que sufren isquemia. Cuando este evento se prolonga se produce una lesión celular que será irreversible (necrosis - infarto). Asociado a las complicaciones que se presentan en el sitio inicial de la obstrucción, también se pueden encontrar las complicaciones a distancia generadas por los émbolos que viajan por el sistema circulatorio arterial o venoso llegando a alojarse con mayor frecuencia en el sistema vascular pulmonar que actúa como un filtro que atrapa al émbolo causando el cuadro clínico conocido como tromboembolia pulmonar $(7,8)$.

La tendencia a sufrir una trombosis puede ser secundaria a un fenómeno llamado trombofilia, situación en la que la homeostasis no está activa y la resistencia a la trombosis está disminuida. Este estado trombofílico puede estar ligado a patrones genéticos como la mutación G2021A de la protrombina y la mutación metilentetrahidrofolato reductasa (MTHFR) responsable de la hiperhomocisteinemia (8). 
Así mismo, se encuentran alteraciones propias de los factores del sistema de coagulación, las cuales son reconocidas como:

- Trombofilia primaria: se puede encontrar aumento en la concentración del factor VIII de la coagulación y deficiencia de antitrombina, proteína $\mathrm{C}$, proteína $\mathrm{S}$, plasminógeno, cofactor II de la heparina y/o factor XII (9).

- Trombofilia secundaria: en esta se ve implicada la irregularidad de otros sistemas como en los casos de neoplasias, insuficiencia cardiaca, insuficiencia respiratoria, ventilación mecánica, inmovilización prolongada, cirugía, embarazo, puerperio, uso de anticonceptivos hormonales, terapia de reemplazo hormonal, arterioesclerosis, dispositivos intravasculares, anticoagulante lúpico y quimioterapia entre otros factores que conllevan aumento de factores de la coagulación VII, VIII, II, fibrinógeno o factor de Von Willebrand (8) manifestándose con una activación desbordada de la homeostasis que sobrecarga los mecanismos de regulación.

Cuando se genera la lesión endotelial se activan los sistemas de coagulación por diversas interrelaciones complejas, entre ellas:

- Flujo sanguíneo: es uno de los principales mecanismos que influyen en la anticoagulación gracias a que el movimiento continuo de la sangre evita la acumulación de factores hemostáticos (8) y plaquetarios activados en un sitio específico (9).

- Pared vascular: en los vasos arteriales la sangre circula a gran velocidad y es sometida a presiones muy altas; los trombos se generan donde predomina la formación de placas ateromatosas o zonas de turbulencia como las bifurcaciones. En los vasos venosos, la turbulencia se genera en las válvulas donde ocurre estasis, baja velocidad y baja presión, generando la activación plaquetaria (9).

- Sistema de coagulación: existe un desequilibrio entre los anticoagulantes naturales y los fibrinolíticos, que conduce a la hipercoagulabilidad (10).

\section{Importancia clínica y factores de riesgo}

La enfermedad tromboembólica venosa es la causa de muerte evitable más importante en el ámbito hospitalario, sin embargo, a pesar de las alternativas terapéuticas con las que se cuenta en la actualidad, esta constituye un riesgo y una complicación para muchos pacientes; incluso se reportan muertes súbitas en las primeras dos horas antes de que el tratamiento pueda ser instaurado de modo efectivo. El estudio multinacional ENDORSE concluyó que la prevención solo se utiliza en el 58,5\% de los pacientes quirúrgicos hospitalizados y en el $39,5 \%$ de los pacientes médicos hospitalizados y hasta en el $80 \%$ de los casos existen más de tres factores de riesgo tromboembólico. Adicionalmente, demostró que la trombosis venosa es un problema de salud pública, condición que puede ser fácilmente modificable, y acarrear una disminución de morbilidad y mortalidad en pacientes hospitalizados para enfermedad quirúrgica y condiciones médicas agudas. Como consideración especial se tiene en cuenta que en este estudio se incluyó población colombiana y se obtuvieron resultados homogéneos respecto a otros países, demostrándose así la subutilización de la tromboprofilaxis en estos pacientes (11).

En consecuencia, el grupo de Barbar et al., desarrolló y validó la escala de Padua (12), la cual permite identificar a los pacientes médicos de alto riesgo embólico y así disponer de un estándar de profilaxis para ellos (tabla 1).

En pacientes con puntajes iguales o menores de tres se indicará tratamiento no farmacológico, en tanto que para puntajes iguales o mayores de cuatro se indicará profilaxis farmacológica (13).

En cuanto a los pacientes en el contexto quirúrgico (postoperatorio), Caprini (14) evidenció de igual forma un aumento en la incidencia de complicaciones secundarias a trombosis venosa, desarrollando consecuentemente en estos un score que determina el riesgo de padecerlas $(15,16)$ (tabla 2$)$.

De igual forma, este score ha sido validado en diferentes tipos de cirugías de acuerdo con los trabajos de Panucci et al. (17). 
Tabla 1. Variables de Padua score. La escala se determina como bajo riesgo un valor $<3$, riesgo alto 4 o más (12).

\begin{tabular}{|c|c|}
\hline \multicolumn{2}{|l|}{ Escala de Padua } \\
\hline Factor de riesgo & Puntuación \\
\hline Cáncer activo & 3 \\
\hline $\begin{array}{l}\text { TEV previo (excluyendo trombosis } \\
\text { superficial) }\end{array}$ & 3 \\
\hline Movilidad reducida (más de 3 días) & 3 \\
\hline $\begin{array}{l}\text { Conocimiento de una condición } \\
\text { trombofílica }\end{array}$ & 3 \\
\hline $\begin{array}{l}\text { Trauma o cirugía reciente (menos de } 1 \\
\text { mes) }\end{array}$ & 2 \\
\hline Ancianos > 70 años & 1 \\
\hline Falla cardíaca o falla respiratoria & 1 \\
\hline $\begin{array}{l}\text { Infarto agudo al miocardio o ataque } \\
\text { cerebrovascular }\end{array}$ & 1 \\
\hline $\begin{array}{l}\text { Infección aguda y/o desorden } \\
\text { reumatológico }\end{array}$ & 1 \\
\hline Obesidad IMC > 30 & 1 \\
\hline Tratamiento hormonal en curso & 1 \\
\hline
\end{tabular}

\section{Tratamiento}

Las guías de práctica clínica basadas en la evidencia, del Colegio americano de cirujanos de tórax, ha desarrollado diferentes escalas, dosis, horarios y manejo de anticoagulantes y las varias alternativas que deben ser utilizadas según la indicación para cada paciente (18).

\section{Formas de prevención}

Todo se fundamenta en tres pilares de acción: ser consciente, ser proactivo y ser profiláctico; la aplicación de medidas profilácticas según el contexto disminuirá la morbilidad y la mortalidad asociada a la enfermedad tromboembólica venosa. Los métodos de profilaxis que han demostrado efectividad en ensayos clínicos controlados pueden ser mecánicos (medias de compresión gradual, compresión neumática intermitente) o farmacológicos (heparinas, hirudina, fondaparinux, anticoagulantes orales).
Además, para todos los pacientes hospitalizados es recomendable la deambulación precoz durante su estancia hospitalaria. La posición de Trendelemburg es una medida eficaz para mejorar el retorno venoso en pacientes aislados o con limitaciones del movimiento. También es importante la hidratación adecuada para prevenir la hemoconcentración (12).

\section{Métodos mecánicos}

Intentan prevenir o reducir la estasis circulatoria y aumentar el flujo venoso en las extremidades inferiores sin riesgo de sangrado. En general, son menos eficaces que los tratamientos farmacológicos. En una revisión sistemática, los métodos mecánicos redujeron cerca de dos tercios el riesgo de trombosis venosa profunda cuando se utilizaron en monoterapia, y hasta un 50\% adicional cuando se añadieron a los métodos farmacológicos (13). Sus contraindicaciones más importantes incluyen: edema masivo, edema pulmonar, arteriopatía periférica grave, neuropatía periférica grave, deformidad importante de la extremidad y dermatitis.

\section{Medias elásticas de compresión gradual}

Son útiles en los pacientes de bajo riesgo. En los individuos de riesgo moderado y alto se pueden asociar a la profilaxis farmacológica. Los resultados de una revisión Cochrane muestran que éstas disminuyeron la incidencia de trombosis venosa profunda en pacientes médicos y quirúrgicos, tanto si se utilizaban solas como asociadas a otros métodos de profilaxis (14). Para profilaxis de la trombosis venosa profunda se aconseja el uso de medias de compresión de clase II (presión al tobillo 23-32 mm Hg). En el mercado se dispone de medias de este tipo hasta por debajo de la rodilla y hasta la ingle. En los pocos estudios disponibles, no hay pruebas claras de un mayor efecto benéfico de las medias por encima la rodilla en relación con las de por debajo de la misma; en todo caso, hay cierta preferencia por las medias por encima de la rodilla.

Se tendrán en cuenta las siguientes precauciones:

- Seleccionar las dimensiones adecuadas de la media.

- Ponerla correctamente con un buen alineamiento. 
Tabla 2. Escala de Caprini (14).

\begin{tabular}{|c|c|}
\hline \multicolumn{2}{|c|}{ Escala de Caprini } \\
\hline Cada factor de riesgo: 1 punto & Cada factor de riesgo: 2 puntos \\
\hline Edad de 40 a 60 años & Edad de 61 a 74 años \\
\hline Antecedente de cirugía mayor en las últimas 4 semanas & Cirugía mayor (1 hora) \\
\hline Várices de miembros inferiores & Artroscopia (> 1 hora) \\
\hline Edema de miembros inferiores & Cirugía laparoscópica (> 1 hora) \\
\hline Enfermedad inflamatoria intestinal & Antecedentes de enfermedad maligna \\
\hline Obesidad IMC > 30 & Vía venosa central \\
\hline $\mathrm{IAM}<1$ mes & Obesidad mórbida IMC > 40 \\
\hline Insuficiencia cardíaca $<1$ mes & Sólo en mujeres: 1 punto \\
\hline Sepsis $<1$ mes & ACO o terapia de reemplazo hormonal \\
\hline Embolia pulmonar OC & Embarazo o posparto menor a 1 mes \\
\hline I. Respiratoria, Neumonía grave $<1$ mes & AGO: pérdida recurrente \\
\hline Inmovilización por férula o yeso & Restricción del crecimiento intrauterino \\
\hline Cada factor de riesgo: 3 puntos & AP: Síndrome antifosfolípidos del embarazo \\
\hline Edad mayor a 75 años & Decolamiento placentario \\
\hline Cirugía mayor de 2 a 3 horas & Síndrome HELLP \\
\hline Antecedente de tromboembolia venosa & Cada factor de riesgo: 5 puntos \\
\hline $\begin{array}{l}\text { Historia de trombosis (tromboembolia pulmonar, trombosis } \\
\text { venosa profunda) }\end{array}$ & Recambio de cadera y rodilla \\
\hline Cáncer activo & Fractura de cadera o pelvis (< 1 mes) \\
\hline Trombofilia congénita o adquirida & Ataque cerebrovascular (<1mes) \\
\hline Síndrome postrombótico & Politrauma (< 1 mes) \\
\hline Obesidad IMC > 50 & Lesión de médula espinal \\
\hline Cirugía mayor que dura $>3$ horas & \\
\hline
\end{tabular}

- Enseñar al paciente como se colocan en forma correcta.

- Retirar la media cada día durante al menos 30 minutos.

- Vigilar de manera periódica la circunferencia de la extremidad.

La compresión neumática intermitente (CPI) es una medida profiláctica eficaz en los pacientes de riesgo moderado o alto con la ventaja de no incrementar el riesgo de sangrado. Puede ser de elección en pacientes de riesgo moderado y contraindicación de anticoagulación (ictus hemorrágico, cirugía intracraneal, cirugía oftálmica o cualquier tipo de cirugía en la que sea previsible un sangrado excesivo). En un metanálisis, los sistemas de compresión neumática intermitente redujeron la incidencia de trombosis venosa profunda en comparación con placebo y medias de compresión gradual solas 
(15). No obstante, se requieren datos comparativos entre las medias de compresión gradual y la compresión neumática intermitente.

En relación con la movilización pasiva, no se dispone de estudios que confirmen su eficacia, pero se puede considerar como medida coadyuvante o alternativa.

Los métodos mecánicos de profilaxis se recomiendan en los pacientes con alto riesgo hemorrágico, o bien como complemento de la profilaxis con anticoagulantes, aconsejando garantizar un uso adecuado y un cumplimiento óptimo del dispositivo mecánico.

\section{Medidas farmacológicas}

Múltiples ensayos clínicos han mostrado la eficacia y la seguridad de los métodos farmacológicos en la prevención de la enfermedad tromboembólica venosa. Hoy se dispone de heparinas, fondaparinux, hirudinas, anticoagulantes orales antivitamina $\mathrm{K}$ y nuevos anticoagulantes (dabigatrán, rivaroxabán, etc.).

\section{Heparinas}

Hay dos grandes tipos de heparinas, las no fraccionadas (HNF) y las fraccionadas o heparinas de bajo de peso molecular (HBPM). Las heparinas son mezclas de polisacáridos de distintos pesos moleculares y se obtienen de tejidos animales. Su efecto deriva de la unión a la antitrombina, inhibidor fisiológico de la coagulación, que inactiva la trombina (IIa), el factor $\mathrm{X}$ y otros factores de la coagulación. Las HBPM se obtienen a partir de la fragmentación de las HNF. Sus características farmacológicas fundamentales, que las diferencian de las HNF, son la mayor capacidad de inhibición del factor X (unas cuatro veces mayor), la unión más débil a las proteínas plasmáticas, la semivida de eliminación más prolongada ( 2 a 4 horas), y la mayor biodisponibilidad por vía subcutánea (superior al 90\%). Como consecuencia, el efecto antitrombótico de las HBPM es más prolongado y con menos variaciones individuales que el de las HNF.

En los pacientes con patología médica existe suficiente evidencia para el uso de cualquiera de las heparinas de bajo peso molecular, resultando ser más costo-efectivo el uso de dalteparina y nadroparina en comparación con la enoxaparina. En general, existe mayor evidencia de la efectividad y seguridad con el uso de la enoxaparina en la tromboprofilaxis temprana de los pacientes de cirugía general, neurocirugía y ortopedia (16); para establecer la dosis, se debe considerar la función renal, la edad, el peso y especialmente el riesgo de hemorragia y en general, no es necesaria la monitorización del efecto anticoagulante (actividad antiXa) en dosis para la profilaxis.

\section{Duración}

La duración de la profilaxis varía según el riesgo de los pacientes y generalmente coincide con la duración del riesgo tromboembólico venoso, según la estimación del médico, normalmente hasta la deambulación del paciente y como mínimo 5-9 días después de la intervención. En la actualidad, se recomienda continuar la tromboprofilaxis con HBPM hasta los 28-35 días después de cirugía de alto riesgo en pacientes postoperados (artroplastia y fractura de rodilla, así como pacientes sometidos a cirugía general de alto riesgo (neoplásica, etc.).

En pacientes de otras cirugías y pacientes médicos, la duración de la profilaxis se extiende mientras persista el riesgo tromboembólico, que en general es de 7 a 10 días. El estudio EXCLAIM propone la continuación de la tromboprofilaxis después del alta en algunos subgrupos de pacientes médicos, como mayores de 75 años, mujeres y pacientes en cama (17).

La tromboprofilaxis con HBPM reduce el riesgo de enfermedad tromboembólica venosa (ETEV) y la mortalidad por tromboembolia pulmonar, y actualmente se consideran de elección, según las principales guías de práctica clínica.

\section{Anticoagulantes orales}

En 2008, la organización "Joint Commission on Accreditation of Healthcare Organizations" incluyó los anticoagulantes orales en la propuesta de los objetivos de seguridad del paciente (National Patients Safety Goals).

Los anticoagulantes antagonistas de la vitamina $\mathrm{K}$ (AVK) actúan impidiendo la carboxilación de los 
factores de la coagulación dependientes de la vitamina $\mathrm{K}$ en los microsomas hepáticos.

Se comercializa el acenocumarol y la warfarina, que se diferencian en sus vidas medias y por tanto en el periodo de acción. Debido a un margen terapéutico muy estrecho, la gran variabilidad individual en el efecto y el riesgo de interacciones con otros medicamentos, no se recomiendan como fármacos de primera línea en la profilaxis de la enfermedad tromboembólica venos, pero se debe considerar su uso en pacientes con alergia o hipersensibilidad a las heparinas y ante síndrome antifosfolípido; su dosificación es variable en torno al International Normalized Ratio (INR, su sigla en inglés).

\section{Sistemas de alarmas}

Debido a que no solo en los pacientes quirúrgicos sino también en los pacientes médicos se reporta en diferentes estudios, una profilaxis antiembólica baja o errónea por una $u$ otra causa, se han diseñado diferentes estrategias para asegurar la profilaxis adecuada en enfermedad tromboembólica venosa, como son el uso de alarmas tanto electrónicas como humanas que adviertan la necesidad de evaluar el riesgo en cada paciente.

Entre las alarmas electrónicas se cuenta con sistemas informáticos que según el nivel de riesgo alertan al clínico sobre la necesidad de profilaxis para enfermedad tromboembólica venosa guiándose en puntuación por condiciones clínicas, diagnósticos actuales y antecedentes. Ejemplos de tales alarmas informáticas y su impacto, se reflejan en la experiencia con un sistema en mención en el Hospital Brigham Women de Boston, que demostró una reducción del $41 \%$ en la enfermedad tromboembólica venosa.

Entre los sistemas de alarma humana están el diseño de tablas de cálculo de riesgo ajustado de forma individual que han demostrado una reducción de hasta un $21 \%$ en la enfermedad tromboembólica venosa.

En el estudio de Kutcher et al. (23), las alertas electrónicas permitieron mejorar de manera significativa la aplicación, tanto de métodos mecánicos, como de los farmacológicos, así como reducir el riesgo de trombosis venosa profunda o tromboembolia pulmonar a los 90 días en un $41 \%$ de los pacientes.

\section{Paciente médico}

Como se mencionó, más del 50\% de los eventos tromboembólicos sintomáticos y $70-80 \%$ de tromboembolia pulmonar mortal corresponden a pacientes no quirúrgicos.

Se han propuesto algunas causas para explicar el porqué de la aplicación subóptima de la tromboprofilaxis. Por un lado, los pacientes médicos constituyen un grupo muy heterogéneo en relación con los factores de riesgo de la enfermedad tromboembólica venosa, motivo por el cual no se dispone de una estratificación en grupos de riesgo tan clara como en los pacientes quirúrgicos. De otra parte, debido a su elevada comorbilidad, el paciente médico tiene más riesgo de hemorragia con la aplicación de tromboprofilaxis farmacológica.

Si bien se dispone de las recomendaciones publicadas por diferentes instituciones (guías) y de los resultados obtenidos en diversos estudios, la aplicación de la tromboprofilaxis en pacientes médicos es baja e inferior a la vista en los pacientes quirúrgicos. Así, en el metanálisis de Dentali et al. se pone de manifiesto que la aplicación de profilaxis farmacológica a pacientes médicos agudos reduce un 57\% el riesgo de tromboembolia pulmonar sintomática, un $62 \%$ el riesgo de tromboembolia pulmonar fatal y un $53 \%$ el riesgo de trombosis venosa profunda sintomática (19).

La tromboprofilaxis es una herramienta de bajo costo que previene eventos agudos como trombosis venosa profunda y tromboembolia pulmonar, que pueden ser causa de muerte en las primeras horas, días y semanas de estancia y después del egreso hospitalario. Cuando el paciente sobrevive a un episodio de tromboembolia puede sufrir secuelas importantes, como hipertensión pulmonar crónica, disminución de la capacidad funcional, deterioro de la calidad de vida, síndrome postflebítico crónico y disminución de la supervivencia. 
En consideración a lo anterior, en 2006 el grupo ARTEMIS22 publicó los resultados de un ensayo clínico aleatorizado en ancianos tras 15 días de seguimiento, que concluyó que una tromboprofilaxis adecuada no incrementa la incidencia de hemorragias cuando se prescribe en forma correcta. Por otro lado, el estudio ENDORSE, de tipo transversal multicéntrico y multinacional, realizado en 358 hospitales de 32 países, que incluyó una población de 68.183 pacientes tanto quirúrgicos como médicos (medicina interna, oncología), consideró que los pacientes con enfermedades crónicas y con reducción de la movilidad se benefician de pautas más largas de tromboprofilaxis.

\section{Incumplimiento de la profilaxis}

Las causas de bajo cumplimiento reportadas en un estudio colombiano y extrapolables a cualquier medio en orden decreciente de frecuencia son, olvido (35\%) error en dosis/tiempos $(25 \%)$, paciente terminal, temor al sangrado $(6 \%)$, hemorragia $(6 \%)$ $\mathrm{y}$ falsa percepción de bajo riesgo (13\%); como se aprecia, el olvido de la prescripción de profilaxis seguida por error en la dosis o en los tiempos de utilización de la misma son las causas más frecuentes, lo cual cobra importancia para la implementación de sistemas de alarmas, retroalimentación y educación continua, sin menospreciar la percepción equívoca de bajo riesgo, con lo que se infiere desconocimiento por parte del clínico del impacto de los factores de riego sobre la enfermedad tromboembólica venosa, además de tener en cuenta que la hemorragia no contraindica métodos mecánicos de profilaxis..

\section{Materiales y métodos, estudio tromboprofilaxis}

Se trató de un estudio descriptivo retrospectivo tipo serie de casos, llevado a cabo durante el período de enero a diciembre del 2014, en el que se tomaron datos de pacientes hospitalizados en el área clínica y quirúrgica de la institución. Se incluyeron variables demográficas y del estado de salud, diagnóstico médico o quirúrgico, tipo de tromboprofilaxis recibida y complicaciones presentadas con seguimiento hasta el alta hospitalaria.

\section{Población y muestra}

El universo estuvo conformado por todos los pacientes que requirieron hospitalización por causa médica y/o quirúrgica en el servicio de urgencias, durante enero a diciembre de 2014.

Se incluyeron pacientes mayores de 18 años en los servicios médicos y quirúrgicos de la institución, registrándose el riesgo de tromboembolia venosa cual evaluado de acuerdo con las guías ACCP (American College of Chest Physicians), en las que se recomienda tromboprofilaxis farmacológica en pacientes con escala de PADUA mayor a 4 que no tengan contraindicaciones para la misma. Se registró el tipo de tromboprofilaxis iniciada en cada paciente, teniendo presente que el uso adecuado de tromboprofilaxis está en relación con la disminución significativa del riesgo de tromboembolia venosa. No se consideró dosis ni duración de la terapia dada la naturaleza del estudio. Se registró la presencia de efectos adversos y complicaciones derivadas de la profilaxis.

\section{Criterios de inclusión}

Pacientes mayores de 18 años, hospitalizados en los servicios de urgencias y hospitalización por más de 48 horas, con diagnóstico principal médico y/o quirúrgico, sin contraindicación de anticoagulación profiláctica o sangrado activo al ingreso.

\section{Criterios de exclusión}

Antecedente de enfermedad oncológica en los últimos seis meses, sangrado moderado por diferentes causas durante su hospitalización, antecedente de enfermedad renal o hepática moderada o severa, alteración hematológica conocida, inicio de síntomas o signos de tromboembolia venosa dentro de las primera 72 horas del ingreso, uso de medicamentos antitrombóticos dentro de la última semana antes del ingreso, sangrado activo al momento del ingreso y conteo de plaquetas menor a 50.000 .

\section{Análisis de los datos}

Se diseñó una base para captura de la información. Se realizó revisión de historias clínicas y 
recolección de información, a cargo del equipo de investigación, conservando el anonimato del paciente. A cada caso se le asignó un código numérico. La información del cuestionario y de los formularios de recolección de los datos se transfirió a una base de datos informática. La base de datos se llevó en EXCEL. Se utilizó el programa SPSS versión 23; las variables cuantitativas se describieron como promedios y rangos, en tanto que las variables categóricas se describieron como frecuencias. Se diseñaron tablas cruzadas para comparar variables categóricas en relación con episodios de sangrado o embólicos y tipo de tromboprofilaxis . El estudio fue aprobado por el comité de ética institucional.

\section{Resultados}

Durante enero a diciembre de 2014 se incluyeron 192 pacientes, 105 (54,7\%) fueron mujeres, con promedio de edad de 59,9 años (rango entre 18 a 89). El 59,9\% de los pacientes cumplía criterios de alto riesgo embólico; el principal medicamento utilizado para tromboprofilaxis fue la enoxaparina en el $67 \%$ de los casos; $7,8 \%$ de los pacientes recibían anticoagulación. El mayor porcentaje de pacientes fue médico (94\%) (tabla 3).

La tabla 4 resume el número de pacientes con tromboprofilaxis acorde con el grupo de riesgo. Pese a tener bajo riesgo, $69 \%$ del total de este grupo recibía algún tipo de tromboprofilaxis; del grupo de alto riesgo 106 (92\%) recibía tromboprofilaxis, de este grupo 14 pacientes estaban anticoagulados.

La tabla 5 describe en detalle el tipo de medicamento utilizado acorde con cada grupo de riesgo.

La tasa de eventos adversos asociada a la terapia fue baja; en un paciente se documentó un episodio tromboembólico (recibía tromboprofilaxis con heparina no fraccionada), en 2 se registró por lo menos un episodio de sangrado, 1 de ellos no recibía tromboprofilaxis, el otro recibía enoxaparina. De los 4 pacientes que requirieron transfusión de algún hemoderivado, 1 no tenía tromboprofilaxis y 2 recibían enoxaparina (20).

La tabla 6 resume las complicaciones derivadas del uso de terapias antitrombóticas.
Tabla 3. Características de la población $n=192$.

\begin{tabular}{|c|c|c|}
\hline & $\mathbf{N}$ & $\%$ \\
\hline $\begin{array}{l}\text { Género femenino/ } \\
\text { masculino }\end{array}$ & $105 / 87$ & $54 / 46$ \\
\hline Edad promedio & 59,9 & Rango 18 a 89 \\
\hline \multicolumn{3}{|l|}{ Tipo de paciente } \\
\hline Médico & 182 & 95 \\
\hline Quirúrgico & 10 & 52 \\
\hline $\begin{array}{l}\text { Osteosíntesis de } \\
\text { cadera }\end{array}$ & 3 & 1,6 \\
\hline Otros & 7 & $3,6 \%$ \\
\hline Paciente crítico & 9 & 47 \\
\hline \multicolumn{3}{|l|}{ Riesgo embólico } \\
\hline Bajo & 39 & 20,3 \\
\hline Moderado & 38 & 19,8 \\
\hline Alto & 115 & 59,9 \\
\hline $\begin{array}{l}\text { Recibió } \\
\text { tromboprofilaxis }\end{array}$ & 149 & 77,6 \\
\hline Enoxaparina & 129 & 67 \\
\hline $\begin{array}{l}\text { Heparina no } \\
\text { fraccionada }\end{array}$ & 20 & 10,4 \\
\hline $\begin{array}{l}\text { Pacientes } \\
\text { anticoagulados }\end{array}$ & 15 & 7,8 \\
\hline
\end{tabular}

Tabla 4. Tromboprofilaxis acorde con grupo de riesgo.

\begin{tabular}{|lc|}
\hline \multicolumn{1}{|c|}{ Riesgo (n) } & $\begin{array}{c}\text { Tratamiento } \\
\text { antitrombótico } \mathbf{n ~ ( \% ) ~}\end{array}$ \\
\hline Bajo (39) & $27(69 \%)$ \\
Moderado (38) & $31(81 \%)$ \\
Alto (115) & $106(92 \%)$ \\
\hline
\end{tabular}

En esta serie de casos no se documentaron medidas no farmacológicas como estrategia de prevención tromboembólica.

\section{Discusión}

A pesar de que existen múltiples estudios que nos dan información acerca de la estratificación adecuada de riesgo de enfermedad tromboembólica y en la forma como se hace el manejo profiláctico de 
Tabla 5. Tipo de tromboprofilaxis.

\begin{tabular}{|lc|}
\hline \multicolumn{1}{|c}{ Riesgo al ingreso } & $\begin{array}{c}\text { Tipo de tromboprofilaxis } \\
\mathbf{n}(\%)\end{array}$ \\
Bajo (39) & $12(30)$ \\
Ninguno & $25(64 \%)$ \\
Enoxaparina & $1(2,5 \%)$ \\
Heparina no fraccionada & $1(2,5 \%)$ \\
Anticoagulación & \\
Moderado (38) & $7(18 \%)$ \\
Ninguno & $28(73 \%)$ \\
Enoxaparina & $3(7,8 \%)$ \\
Heparina no fraccionada & $0(0)$ \\
Anticoagulación & \\
\hline Alto (115) & $28(24 \%)$ \\
Ninguno & $76(66 \%)$ \\
\hline Enoxaparina & $16(13 \%)$ \\
Heparina no fraccionada & $14(12 \%)$ \\
\hline Anticoagulación & \\
\hline
\end{tabular}

Tabla 6. Complicaciones.

\begin{tabular}{|lcr|}
\hline & $\mathbf{n}$ & $\%$ \\
Episodios embólicos & 1 & 0,5 \\
Sangrado & 2 & 1 \\
$\begin{array}{l}\text { Transfusión } \\
\text { hemoderivados }\end{array}$ & 4 & 2,1 \\
\hline
\end{tabular}

la misma, se debe tener en cuenta la forma de recolección de la información pues la mayoría de los estudios son extraídos de historias clínicas y no directamente del paciente.

Este estudio muestra que en la institución donde se llevó a cabo, el mayor número de pacientes pertenece al grupo de pacientes con causa médica, similar al de otras poblaciones hospitalizadas con un promedio de $95 \%$, datos que difieren de los encontrados para Colombia en el estudio IMPROVE (76\%), el cual incluyó una muestra de 1.948 pacientes del área médica, hace tres años, que representan el 64\%).
El 54,7\% de los pacientes incluidos fueron mujeres, con promedio de edad de 59,9 años. El 59,9\% de los pacientes cumplían criterios de alto riesgo embólico, por el grupo de edad con población adulta mayor además de tener gran cantidad de comorbilidades; a pesar de tener bajo riesgo, 69\% del total de este grupo venían recibiendo algún tipo de tromboprofilaxis. Del grupo de alto riesgo, 106 (92\%) recibían tromboprofilaxis, a pesar de que ENDORSE demuestra que la profilaxis apropiada en pacientes en riesgo que se beneficiarían de ella; sólo se llevó a cabo en aproximadamente la mitad de los evaluados.

El principal medicamento utilizado para tromboprofilaxis fue la enoxaparina en el $67 \%$ de los casos; $7,8 \%$ de los pacientes recibían anticoagulación (20).

Los resultados de este estudio difieren con los encontrados en otras series (11), hecho que probablemente se deba a la difusión de la información y el tiempo dedicado a desarrollar, implementar y seguir las guías de práctica clínica que pueden haber sido insuficientes para garantizar un cambio importante en los hábitos de prevención de la tromboembolia venosa en el área médica en Colombia.

El diseño del estudio puede tener limitaciones pues sólo brinda información sobre el perfil de riesgo del paciente y la pertinencia de la profilaxis hasta el momento de la evaluación; no se pueden hacer inferencias sobre el perfil de riesgo y lo apropiado de la profilaxis posteriormente en la hospitalización del sujeto, además por el corto periodo de recolección de la muestra; sin embargo ofrece información acerca del perfil de los pacientes y de esta forma sensibiliza a las instituciones.

\section{Conclusiones}

Existen múltiples formas de profilaxis, desde las no farmacológicas hasta las farmacológicas y combinadas según el contexto del paciente con alto perfil de seguridad y eficacia y bajos costos, pero con bajo nivel de adherencia según múltiples estudios dentro de los cuales sobresale el ENDORSE, en donde se halla sin excepción a nivel mundial en 358 hospitales en 32 países que la profilaxis aun en pacientes de riego solo se realiza en el $56,7 \%$. 
Distintas organizaciones han promovido guías, protocolos y distintas estrategias para mejorar la tromboprofilaxis (21). Las guías de la ACCP recomiendan que cada hospital desarrolle sus estrategias para la valoración del riesgo de la enfermedad tromboembólica venosa y la implementación de la tromboprofilaxis apropiada y además que se utilicen también en formato impreso.

Además de las distintas formas de profilaxis la organización "Joint Commission on Accreditation of Healthcare Organizations" incluyó los anticoagulantes orales en la propuesta de los objetivos de seguridad del paciente (National Patients Safety Goals).

Parece ser que la principal causa de incumplimiento de la tromboprofilaxis es el olvido y conocimiento insuficiente por parte del personal en salud - médicos, de los factores de riesgo de la enfermedad tromboembólica venosa y su valoración. Se han propuesto distintas estrategias de calidad para mejorar la aplicación de la tromboprofilaxis: educación a los clínicos mediante sesiones, diseminación de guías, protocolos, documentos impresos, sistemas de alarma, entre otros; además de sistemas recordatorios como afiches y soporte a la prescripción, auditorías y feedback, educación al paciente; cambios organizativos e incentivos económicos y políticos en los cuales se divulguen formas de profilaxis, pertinencia y valoración del riesgo individual.

Parece ser que la tromboprofilaxis mejoró de manera significativa después de la aplicación de programas de educación, como sesiones informativas y documentos impresos (43\% antes del programa y 58\% después) y que la diseminación pasiva de las guías de práctica clínica (GPC) es el método con menor tasa de seguimiento.

Por otro lado, las estrategias proactivas como las alertas electrónicas, recordatorios junto con auditorías y feedback son más efectivas. En el estudio de Kutcher et al. Las alertas electrónicas permitieron mejorar significativamente la aplicación tanto de los métodos mecánicos como de los farmacológicos, así como reducir el riesgo de trombosis venosa profunda o tromboembolia pulmonar a los 90 días en un $41 \%$. Finalmente, la incorporación de múltiples estrategias (programas educativos, herramientas de soporte a la prescripción, recordatorios y alertas, auditorías y feedback) son más efectivos para mejorar la tromboprofilaxis que la aplicación de estrategias en forma aislada. Las guías de la ACCP también recomiendan el uso de estrategias proactivas.

En los hospitales se tiene el potencial de reducir la carga del costo nacional e individual de enfermedad tromboembólica venosa y cumplir con mejores expectativas de calidad, garantizando que los eventos tromboembólicos venosos se prevengan mediante profilaxis con el uso de las opciones ampliamente disponibles en las guías basadas en la evidencia, según identificación de pacientes con riesgo de enfermedad tromboembólica venosa.

En los pacientes con patología médica y/o quirúrgica con bajo a moderado riesgo las medidas no farmacológicas con bajos costos han demostrado alta seguridad y efectividad, mientras que en pacientes de alto riesgo como complemento a las medidas farmacológicas han disminuido hasta un 50\% adicional la casuística de la enfermedad tromboembólica venosa. Por otra parte, existe suficiente evidencia para el uso de cualquiera de las heparinas de bajo peso molecular con adecuado nivel de seguridad, eficacia y costo respecto de la no tromboprofilaxis con todo el costo derivado de ello, como lo son el tratamiento de la enfermedad tromboembólica venosa y sus posibles complicaciones, secuelas indemnizaciones e implicaciones sociales.

\section{Bibliografía}

1. Bagot CN, Arya R. Virchow and his triad: a question of attribution. Br J Haematol. 2008;143:180-90.

2. Virchow RC. Cellular pathology as based upon physiological and pathological history. [Tr. from the 2 nd. ed. of the original by Frank Chance]. Birmingham; Gryphod Editions, Ltd; 1978.

3. Dickson BC. Venous thrombosis: on the history of Virchow's Triad. Univ Toronto Med J. 2004;81:166-71.

4. Geerts WH, Pineo GF, Heit JA, et al. Prevention of venous thromboembolism: the Seventh ACCP Conference on Antithrombotic and Thrombolytic Therapy. Chest 2004;126(3 suppl):338S-400S.

5. Yamada N, Hanzawa K, Ota S, et al. Occurrence of Deep Vein Thrombosis among Hospitalized Non-Surgical Japanese Patients. Ann Vasc Dis. 2015;8(3):203-9. 
6. Sandler DA, Martin JF. Autopsy proven pulmonary embolism in hospital patients: are we detecting enough deep vein thrombosis? J R Soc Med 1989;82(4):203-5.

7. Ashish J. The research priority setting working group of the World alliance for Patient safety. Summary of the evidence on patient safety: implications for research. World Health Organization; 2008.

8. Abraham Majluf, Espinosa F. Fisiopatología de la trombosis. Medigraphic Anestesia. Gac Med Mexico; 2007.

9. Lobato-Mendizábal E, Majluf-Cruz A. Thrombophilia, thromboembolism and the use of the unfractionated and low-molecular-weight heparins. Rev Invest Clin. 2000;52:529-45.

10. Martínez-Murillo C, Quintana-González S, Ambriz-Fernández R, Hernández Paula M. El problema trombótico. Hematología. 2000;1:17-20.

11. Cohen AT, Tapson VF, Bergmann JF, Goldhaber SZ, Kakkar AK, Deslandes B, et al. Venous thromboembolism risk and prophylaxis in the acute hospital care setting (ENDORSE study): a multinational cross-sectional study. Lancet. 2008;371:387-94.

12. Barbar S, Noventa F, Rossetto V, Ferrari A, Brandolin B. A risk assessment model for the identification of hospitalized medical patients at risk for venous thromboembolism: the Padua Prediction Score. J Thromb Haemost. 2010;8(11):2450-7.

13. Caprini JA, Arcelus JI, Hasty JH, Tamhane AC, Fabrega F. Thrombosis risk assessment as a guide to quality patient care. Dis Mon. 2005;51(1-3):70-8.

14. Caprini JA. Thrombosis risk assessment as a guide to quality patient care. Semin Thromb Hemost. 1991;17(Suppl 3):304-12.
15. Rogers SO Jr, Kilaru RK, Hosokawa P, Henderson WG, Zinner MJ, Khuri SF. Multivariable predictors of postoperative venous thromboembolic events after general and vascular surgery: results from the patient safety in surgery study. J Am Coll Surg. 2007;204(6):1211-21.

16. Bahl V, Hu HM, Henke PK, Wakefield TW, Campbell DA Jr,A validation study of a retrospective venous thromboembolism risk scoring method. Ann Surg. 2010;251(2):344-50.

17. Pannucci CJ, Bailey SH, Dreszer G, Fisher Wachtman C, Zumsteg JW et al Validation of the Caprini risk assessment model in plastic and reconstructive surgery patients. J Am Coll Surg. 2011;212(1):105-12.

18. Guyatt GH, Akl EA, Gutterman DD, Schunemann HJ. Antithrombotic therapy and prevention of thrombosis, 9th. ed: American College of Chest Physicians Evidence-Based Clinical Practice Guidelines. CHEST. 2012;141(2 suppl)7S-47S. doi: $10.1378 /$ chest.1412S3.

19. Bick RL. Proficient and cost-effective approaches for the prevention and treatment of venous thrombosis and thromboembolism. Drugs. 2000;60(3):575-95.

20. Gould MK, Debitzer AD, Doyle RL, Hastie TJ, Garber AM. Low-molecular-weight heparins compared with unfractionated heparin for treatment of acute vein thrombosis. A metaanalysis of randomized, controlled trials. Ann Intern Med. 1999; 130:800-9.

21. Cohen AT, Davidson BL, Gallus AS, Lassen MR, Prins MH, Tomkowski W. Efficacy and safety of fondaparinux for the prevention of venous thromboembolism in older acute medical patients: randomised placebo controlled trial. BMJ. 2006;332:325-9. 